Journal of Patient-Centered

\title{
Team-Based Care and Patient Satisfaction in the Hospital Setting: A Systematic Review
}

\author{
Kristen K. Will \\ Melissa L. Johnson \\ Gerri Lamb
}

Follow this and additional works at: https://aah.org/jpcrr

Part of the Health and Medical Administration Commons, Health Services Administration Commons, Health Services Research Commons, and the Other Medical Specialties Commons

\section{Recommended Citation}

Will KK, Johnson ML, Lamb G. Team-based care and patient satisfaction in the hospital setting: a systematic review. J Patient Cent Res Rev. 2019;6:158-71. doi: 10.17294/2330-0698.1695

Published quarterly by Midwest-based health system Advocate Aurora Health and indexed in PubMed Central, the Journal of Patient-Centered Research and Reviews (JPCRR) is an open access, peer-reviewed medical journal focused on disseminating scholarly works devoted to improving patient-centered care practices, health outcomes, and the patient experience. 


\title{
Team-Based Care and Patient Satisfaction in the Hospital Setting: A Systematic Review
}

\author{
Kristen K. Will, MHPE, PA-C, ${ }^{1}$ Melissa L. Johnson, MSLS, ${ }^{2}$ Gerri Lamb, PhD, RN ${ }^{3}$ \\ ${ }^{1}$ College of Health Solutions, Arizona State University, Phoenix, AZ; ${ }^{2}$ Library, Arizona State University, Phoenix, AZ; \\ ${ }^{3}$ Center for Advancing Interprofessional Practice, Education and Research, Edson College of Nursing and Health \\ Innovation, Arizona State University, Phoenix, AZ
}
Purpose Limited research examining the relationship between team-based models of care and patient satisfaction in the hospital setting is available. The purpose of this literature review was to explore this relationship as well as the relationships between team composition, team-based interventions, patient satisfaction, and other outcomes of care when measured as part of the study.

Methods A systematic appraisal of research studies published through February 2017 was conducted using PubMed, Cochrane Library, CINAHL, Embase, Ovid, gray literature and Google Scholar. Inclusion criteria were 1) experimental (randomized control trials), quasi-experimental, or non-experimental (cross-sectional) study design; 2) team-based care interventions; 3) hospital setting; 4) patient satisfaction measured as an outcome; and 5) published in English.

Results The literature search yielded 15,247 citations. In total, 142 articles were retrieved for full-text screening; 21 studies met inclusion criteria. Overall, $57 \%$ of the studies identified a statistically significant improvement in patient satisfaction associated with team-based care. Team-based care interventions ranged from single team activities such as multidisciplinary rounds to comprehensive team-based models of care. Patient satisfaction scores were greater with teams that had more than two professions and more comprehensive team-based models. About one-quarter of studies that measured patient satisfaction and at least one additional outcome demonstrated improvement in both.

Conclusions Team-based care may positively affect patient satisfaction. Team composition and type of team intervention appears to influence the strength of the relationship. Improvements in satisfaction are not consistently accompanied by improvements in other outcomes. (J Patient Cent Res Rev. 2019;6:158-171.)

Keywords patient satisfaction; interprofessional collaboration; hospital care; systematic review; quality metrics

$\mathrm{W}$ ith the advent of pay-per-performance and value-based reimbursement in the health care setting, emphasis on new innovative care delivery models is necessary. Health care systems strive to align their care delivery models with the Triple Aim: improved population health, improved patient experience, lower health care costs. ${ }^{1}$ Over the last several decades, growth in team-based models of care have emerged in many different sectors of health care. ${ }^{2}$

Correspondence: Kristen K. Will, MHPE, PA-C, Arizona State University Downtown Campus, 550 N. 3rd Street, Phoenix, AZ 85004 (kkwill@asu.edu)
This growth is largely due to the growing complexity of the health care system and the recognition that no individual health care provider or discipline can provide comprehensive care for patients, especially in the hospital setting. ${ }^{3}$

While many studies have shown a positive relationship between team-based care and patient outcomes, such as reducing cost and improving quality, few examine the impact of the health care team on the patient experience. Furthermore, defining the exact team composition or intervention to promote the Triple Aim also remains uncertain. With the fragmentation of the health care system, team-based care, often 
referred to as interprofessional collaborative practice, is imperative to provide high-value, patient-centered care, and identifying the team-based models of care that promote the largest impact on quality, safety, and patient experience is crucial. ${ }^{4}$

The first of its kind, this review was designed to examine the relationships between team-based models of care and patient satisfaction in the hospital setting utilizing a wide scope of research. A secondary purpose was to explore relationships among team composition, team-based interventions, and patient satisfaction.

\section{Defining Team Composition and Interventions}

The composition and key features of health care teams have been described in several different ways. One of the commonly cited definitions of teams was put forward by the Institute of Medicine's workgroup on "team-based health care." Its definition includes two or more health care professions and often includes the patient and sometimes the patient's family or caregivers. $^{2}$ Additional definitions describe effective teams as those that share common goals and collaborate to deliver high-value, patient-centered care. ${ }^{5}$ The World Health Organization defines interprofessional collaborative practice, a synonym to team-based health care, as multiple health workers from various professional backgrounds working together with patients, families, caregivers, and communities to deliver high-quality patient care. ${ }^{6}$ Team-based care has been further supported through development of interprofessional education and collaborative practice competencies from the World Health Organization and the Interprofessional Education Collaborative. ${ }^{6,7}$

Beyond the definition of team composition in health care, broad categories of team-based care interventions proposed by Reeves et al have been described in the literature: interprofessional coordination, interprofessional collaboration, and interprofessional teamwork. ${ }^{8}$ These categories describe a continuum of team-based care ranging from coordination of care - which includes periodic, ad hoc communication (interprofessional coordination) - to full integration of care, in which teams are designed a priori to provide comprehensive care for a specific patient population (interprofessional teamwork). ${ }^{8}$ To date, no studies have examined the impact of various team composition and/ or team-based care interventions on patient experience.

\section{Evidence for Team-Based Care}

Although team-based care has been well-described and significant advances to team-based care have occurred in recent years, barriers continue to exist. These include lack of reimbursement models, lack of interprofessional training to support interprofessional collaborative practice, cultural differences among health care professions, and lack of understanding of optimal team structure and function. ${ }^{9}$

Despite these barriers, there is a growing body of research that indicates team-based care is associated with improved patient outcomes. Some of the outcomes linked to team intervention include decreased readmission rates to the hospital for highrisk patient populations, decreased adverse events for hospitalized patients, and decreased length of stay in the hospital. ${ }^{2,4,10}$ Similar improved patient outcomes have been associated with team-based models of care in the ambulatory care setting. ${ }^{10,11}$ Patient-centered medical homes (PCMH) using team models have shown improved coordination of care, access to care, and quality and safety metrics. Specifically, in patient populations with chronic comorbid diseases, PCMH models have demonstrated decreased pharmacy expenditures and emergency department visits. ${ }^{12}$

With the growing emphasis on the importance of patient-centered care and the patient experience, there has been renewed interest in the impact of teamwork on patient satisfaction. Teams are being looked to as a vehicle for enhancing patient satisfaction as well as achieving payment incentives in value-based payment models. ${ }^{13}$ Patient satisfaction has been an interest in patient outcome research for decades but has not been linked to direct reimbursement until recently. With the arrival of pay-for-performance and value-based care, patient satisfaction scores may be included in qualitybased payment systems for ambulatory care services and for inpatient care. ${ }^{14}$ While performance on these scores is still attributed to individual providers and systems, there is a growing movement in the United States to recognize the contribution of teams in emerging payment models. ${ }^{15,16}$

Demonstrating clear patient outcomes related to teambased care is imperative to drive further research, health care policy changes, and clinical practice guidelines. Wen and Schulman conducted a systematic 
review of the effect of team-based care models on patient satisfaction. ${ }^{13}$ In this meta-analysis examining only randomized controlled trials conducted through 2012, they found an equivocal relationship between team-based care and patient satisfaction. The authors noted limitations within their review, including a lack of definition for "team," inconsistency between studies describing the team intervention, and wide variation of patient satisfaction measurement tools.

Since that time, the body of knowledge regarding teambased care and team science has greatly expanded ${ }^{16}$ and extends outside the confines of randomized controlled trials. In addition to patient satisfaction team composition, and team-based interventions, the review presented herein will examine a broader scope of team-based care studies, including experimental, quasi-experimental and non-experimental (cross-sectional), allowing for a more comprehensive analysis of the research in this area. Unlike the review performed by Wen and Schulman, ours is specifically focused on the hospital setting, an important difference because many factors that affect patient satisfaction - along with reimbursement structures for team-based care, team composition structures, and team interventions ${ }^{15-17}$ - greatly differ between inpatient and ambulatory care settings. To prepare for new team-based incentive models, it is critical to understand the impact of team-based care on patient satisfaction and to further understand how team composition and which type of team-based interventions truly impact the patient experience.

\section{METHODS}

We adhered to the Preferred Reporting Items for Systematic Reviews and Meta-Analyses (PRISMA) criteria for conducting a systematic search of the literature, which can be found within the PRISMA checklist. ${ }^{18}$ The heterogeneity of the identified study designs precluded performing a meta-analysis. ${ }^{19}$

\section{Inclusion and Exclusion Criteria}

Inclusion criteria for the review were: experimental or quasi-experimental or non-experimental (crosssectional) design; team-based care defined as 2 or more people from at least 2 professions or disciplines working together to provide patient care; patient care took place within any hospital department; and article written in English. International studies were included to allow for comprehensive analysis. Required outcome measures included directly gathered patient satisfaction data from any patient population.

Reviews, expert opinion, background articles, and conference proceedings were excluded. Studies also were excluded if they only described providers' impressions of patient satisfaction or provider satisfaction rather than actual measurements of patient satisfaction.

\section{Search Strategies and Study Selection}

PubMed, Cochrane Library, CINAHL, Embase, Ovid, gray literature, and Google Scholar databases were searched. Database search duties were split between the first and second authors, and searched from inception through February 2017. Reference lists from retrieved articles were also examined for additional articles. Articles retrieved were initially reviewed for relevance by title and abstract for inclusion of interprofessional patient care teams, patient satisfaction, and the hospital setting. After duplicate articles were removed, the remaining articles were analyzed per the inclusion and exclusion criteria within the full text.

In each database search, both Medical Subject Headings $(\mathrm{MeSH})$ and natural language keywords were utilized (Online Appendix 1). To capture the concept of teambased models of care, the following search terms were used: patient care team, interdisciplinary care team, interdisciplinary health team, medical care team, health care team, interprofessional collaboration, and interprofessionalism. "Critical care" and "hospital based care" were used to search for the hospital care setting. Patient satisfaction was searched using the following terms: patient satisfaction, patient satisfaction rate, patientcentered outcome, patient outcomes, patient experience, the Hospital Consumer Assessment of Healthcare Providers and Systems (HCAHPS), Press Ganey surveys, and hospital value-based purchasing. All terms were searched as keywords in the text, title, or abstract.

\section{Data Extraction and Quality Assessment}

Study design, sample population, team composition, team intervention, patient satisfaction assessment tool, and study results were extracted from each article for further analysis using a standard template. The first author extracted initial data, and the second author verified the extracted data to ensure reliability. When a discrepancy on data points was observed, consensus between both reviewers was achieved. 
Team composition was divided into 2 categories: dyad teams ( 2 people from 2 different health professions) and multiprofessional teams (3 or more members representing at least 3 health professions). Data were gathered from study descriptions of who from the health care team participated and placed into one of the categories.

Team-based care interventions were categorized into the 3 categories previously described by Reeves et al as follows:

- Interprofessional coordination - Various individuals representing different health care professions who work together to provide care. The activities, such as communication, are less frequently interprofessional and are not necessarily predetermined as part of regular care. ${ }^{8}$
- Interprofessional collaboration - This type of interprofessional work involves health care professionals who come together on a regular basis to intentionally communicate or make decisions regarding patient care. For example, multidisciplinary rounds can be considered a type of interprofessional collaboration. $^{8}$

- Interprofessional teamwork - Activities of these care teams are fully interdependent, and teams come together with shared goals, an identity, and mental model to deliver integrated care. Care coordination teams are an example of interprofessional teamwork. ${ }^{8}$

The first and second authors collectively reviewed the methods section of articles to place each of the studies into one of the team intervention categories. When there was discrepancy on category, a consensus between the

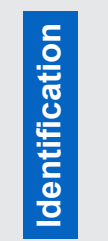

Medline $(n=595)$

Cochrane $(n=92)$

CINAHL $(n=214)$

Gray literature $(n=3)$

Google Scholar $(n=9102)$

Embase $(n=4745)$

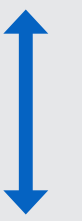

Articles identified as potentially relevant and screened for retrieval $(n=142)$

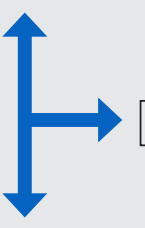

Excluded for duplicates $(n=18)$

Articles reviewed via full text for inclusion criteria $(n=124)$

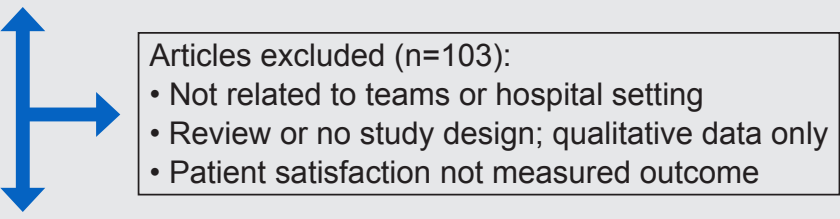

음

Articles included for systematic review $(n=21)$

Figure 1. PRISMA flowchart. 
reviewers was achieved through joint review of the study intervention and category definitions.

The quality of each article was assessed independently by the first and second author using the modified GRADE criteria, which consist of type of evidence, quality, consistency, directness, and effect size. ${ }^{20}$ Quality of articles were rated on a scale from 0 to 4 based on the standardized scale $(0=$ very low; $1=10 w$; 2=moderate; 3=high; 4=very high). Cohen's kappa coefficient was used to estimate the consistency of the individual raters. ${ }^{21}$

\section{RESULTS}

\section{Study Characteristics}

The literature search yielded 15,247 citations. After assessing for relevance, 142 articles were retrieved for full-text screening. After duplicates were removed, 124 articles were screened, with 21 studies subsequently included for analysis based on the inclusion and exclusion criteria (Figure 1).

The 21 reviewed studies were published between 1999 and 2016.22-42 Four studies were randomized controlled trials, 16 were quasi-experimental, and 1 used a crosssectional design (Table 1). The majority of studies were conducted in an academic medical center or tertiary hospital setting $(n=16)$. The remainder occurred in community hospital settings. Patient populations included adult patients in all studies except one, which focused on pediatric patients. ${ }^{29}$

Team composition - multiprofessional or dyad varied among studies (Table 2). The majority of teams were multiprofessional ( $71 \%$ vs $29 \%$ dyad). Most multiprofessional teams included more than 3 professions in the team $(n=15)$. Multiprofessional teams routinely consisted of a combination of physicians, nurses, advance practice providers, social workers, case management, and therapy services. A typical composition of dyads was physician and nurse or advanced practice provider. One study included the patient as a member of the team, while another considered the family or caregiver as part of the team..$^{26,30}$

Type of team-based care intervention also varied. More than half of the studies utilized interprofessional teamwork $(\mathrm{n}=11,52 \%)$ versus interprofessional coordination $(\mathrm{n}=7,33 \%)$ or interprofessional collaboration $(n=3,14 \%)$ (Figure 2$)$.

Quality assessment per GRADE criteria was overall low to moderate due to the limited amount of randomized controlled trials. Interrater reliability, measured through Cohen's kappa, was calculated at $0.87(\mathrm{P}<0.001)$.

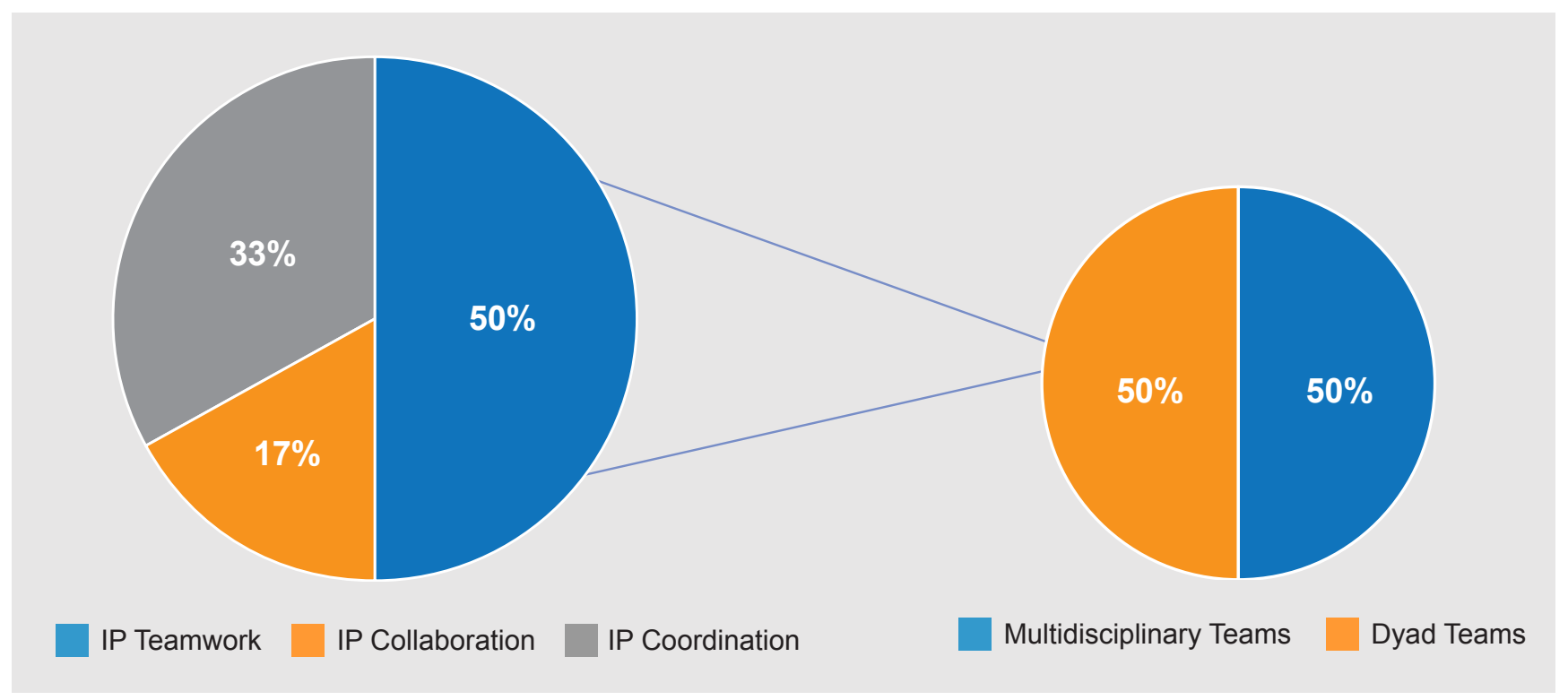

Figure 2. Team intervention type associated with improved patient satisfaction. IP, interprofessional 
Table 1. Study Characteristics

\begin{tabular}{|c|c|c|c|c|c|}
\hline Citation & Study Design & Setting & Participants & $n^{*}$ & Intervention \\
\hline $\begin{array}{l}\text { Ahmed et al } \\
(2010)^{22}\end{array}$ & $\begin{array}{l}\text { quasi- } \\
\text { experimental }\end{array}$ & AMC & $\begin{array}{l}\text { geriatric service } \\
\text { patients }\end{array}$ & $\begin{array}{l}\text { control }=383 \\
\text { intervention }=1064\end{array}$ & Acute Care for Elderly (ACE) team \\
\hline $\begin{array}{l}\text { Arbaje et al } \\
(2014)^{27}\end{array}$ & $\begin{array}{l}\text { quasi- } \\
\text { experimental }\end{array}$ & AMC & $\begin{array}{l}\text { geriatric service } \\
\text { patients }\end{array}$ & $\begin{array}{l}\text { control = } 118 \\
\text { intervention = } 151\end{array}$ & $\begin{array}{l}\text { Geriatric Floating Interdisciplinary } \\
\text { Transition Team (Geri-FITT) dyad }\end{array}$ \\
\hline $\begin{array}{l}\text { Auerbach et } \\
\text { al }(2012)^{30}\end{array}$ & $\begin{array}{l}\text { quasi- } \\
\text { experimental }\end{array}$ & AMC & $\begin{array}{l}\text { general medicine } \\
\text { unit patients }\end{array}$ & 313 & $\begin{array}{l}\text { Triad for Optimal Patient Safety } \\
\text { (TOPS), interprofessional education, } \\
\text { and multidisciplinary team champions }\end{array}$ \\
\hline $\begin{array}{l}\text { DeBehnke } \\
\text { et al }(2002)^{31}\end{array}$ & $\begin{array}{l}\text { quasi- } \\
\text { experimental }\end{array}$ & AMC & $\begin{array}{l}\text { emergency } \\
\text { department patients }\end{array}$ & 454 & Physician-registered nurse dyads \\
\hline $\begin{array}{l}\text { Finch et al } \\
(1999)^{23}\end{array}$ & $\begin{array}{l}\text { quasi- } \\
\text { experimental }\end{array}$ & rural hospital & discharged patients & 121 & Multidisciplinary rounds \\
\hline $\begin{array}{l}\text { Forster et al } \\
(2005)^{25}\end{array}$ & $\begin{array}{l}\text { experimental } \\
\text { (RCT) }\end{array}$ & $\begin{array}{l}\text { AMC } \\
\text { (Canada) }\end{array}$ & $\begin{array}{l}\text { general medicine } \\
\text { unit patients }\end{array}$ & $\begin{array}{l}\text { control }=155 \\
\text { intervention }=135\end{array}$ & $\begin{array}{l}\text { Physician-clinical nurse specialist } \\
\text { dyads }\end{array}$ \\
\hline $\begin{array}{l}\text { Gade et al } \\
(2008)^{28}\end{array}$ & $\begin{array}{l}\text { experimental } \\
\text { (RCT) }\end{array}$ & varied & $\begin{array}{l}\text { palliative care } \\
\text { patients }\end{array}$ & $\begin{array}{l}\text { control }=23 \\
\text { intervention }=275\end{array}$ & Multidisciplinary care team \\
\hline $\begin{array}{l}\text { Hastings et } \\
\text { al }(2016)^{26}\end{array}$ & $\begin{array}{l}\text { quasi- } \\
\text { experimental }\end{array}$ & $\begin{array}{l}\text { AMC } \\
\text { (Canada) }\end{array}$ & $\begin{array}{l}\text { general medical unit } \\
\text { patients }\end{array}$ & $\begin{array}{l}\text { baseline }=26 \\
\text { intervention }=37\end{array}$ & $\begin{array}{l}\text { Interprofessional coordination and } \\
\text { multidisciplinary rounds }\end{array}$ \\
\hline $\begin{array}{l}\text { Hung et al } \\
(2013)^{32}\end{array}$ & $\begin{array}{l}\text { quasi- } \\
\text { experimental }\end{array}$ & $\begin{array}{l}\text { urban tertiary } \\
\text { care hospital }\end{array}$ & $\begin{array}{l}\text { geriatric patients } \\
\text { with acute illness }\end{array}$ & $\begin{array}{l}\text { control }=173 \\
\text { intervention }=17\end{array}$ & $\begin{array}{l}\text { Mobile Acute Care for Elderly (MACE) } \\
\text { unit }\end{array}$ \\
\hline $\begin{array}{l}\text { lannuzzi et } \\
\text { al }(2015)^{33}\end{array}$ & $\begin{array}{l}\text { quasi- } \\
\text { experimental }\end{array}$ & AMC & $\begin{array}{l}\text { general medical unit } \\
\text { patients }\end{array}$ & $\begin{array}{l}\text { resident team }=\sim 562 \\
\text { MLP team }=\sim 289\end{array}$ & $\begin{array}{l}\text { Hospitalist-MLP dyad vs hospitalist- } \\
\text { resident dyad }\end{array}$ \\
\hline $\begin{array}{l}\text { Kane et al } \\
(2016)^{34}\end{array}$ & $\begin{array}{l}\text { quasi- } \\
\text { experimental }\end{array}$ & AMC & $\begin{array}{l}\text { general medical unit } \\
\text { patients }\end{array}$ & not provided & $\begin{array}{l}\text { Team huddles with "playbook" for bed } \\
\text { management and daily multidisciplinary } \\
\text { team report for discharges }\end{array}$ \\
\hline $\begin{array}{l}\text { Kara et al } \\
(2015)^{35}\end{array}$ & $\begin{array}{l}\text { quasi- } \\
\text { experimental }\end{array}$ & AMC & $\begin{array}{l}\text { general medical } \\
\text { unit patients } \\
\text { and surgical unit } \\
\text { patients }\end{array}$ & 110 & Accountable care team \\
\hline $\begin{array}{l}\text { Laird-Fick et } \\
\text { al }(2011)^{36}\end{array}$ & $\begin{array}{l}\text { quasi- } \\
\text { experimental }\end{array}$ & $\begin{array}{l}\text { community } \\
\text { hospital }\end{array}$ & $\begin{array}{l}\text { adult emergency } \\
\text { department patients }\end{array}$ & $\begin{array}{l}\text { control }=81 \\
\text { intervention }=86\end{array}$ & $\begin{array}{l}\text { Interprofessional education and } \\
\text { collaboration }\end{array}$ \\
\hline $\begin{array}{l}\text { San Martin- } \\
\text { Rodriguez et } \\
\text { al }(2008)^{37}\end{array}$ & $\begin{array}{l}\text { non- } \\
\text { experimental } \\
\text { (cross-sectional) } \\
\end{array}$ & AMC (Spain) & $\begin{array}{l}\text { general medical unit } \\
\text { patients with cancer }\end{array}$ & 312 & $\begin{array}{l}\text { Low- vs high-intensity interprofessional } \\
\text { teams }\end{array}$ \\
\hline $\begin{array}{l}\text { Menefee et } \\
\text { al }(2014)^{38}\end{array}$ & $\begin{array}{l}\text { quasi- } \\
\text { experimental }\end{array}$ & rural hospital & $\begin{array}{l}\text { general medical unit } \\
\text { patients }\end{array}$ & 217 & $\begin{array}{l}\text { Menefee model and interdisciplinary } \\
\text { plan of care }\end{array}$ \\
\hline $\begin{array}{l}\text { Preen et al } \\
(2005)^{24}\end{array}$ & $\begin{array}{l}\text { experimental } \\
(\mathrm{RCT})\end{array}$ & $\begin{array}{l}\text { tertiary care } \\
\text { hospital } \\
\text { (Australia) }\end{array}$ & $\begin{array}{l}\text { patients } \\
\text { with chronic } \\
\text { cardiorespiratory } \\
\text { disease } \\
\end{array}$ & 128 & $\begin{array}{l}\text { Multidisciplinary discharge planning } \\
\text { team }\end{array}$ \\
\hline $\begin{array}{l}\text { Roy et al } \\
(2008)^{39}\end{array}$ & $\begin{array}{l}\text { quasi- } \\
\text { experimental }\end{array}$ & AMC & $\begin{array}{l}\text { general medical } \\
\text { service patients }\end{array}$ & $\begin{array}{l}\text { control }=4202 \\
\text { intervention }=992\end{array}$ & Multidisciplinary hospitalist team \\
\hline $\begin{array}{l}\text { Scotten et al } \\
(2015)^{29}\end{array}$ & $\begin{array}{l}\text { quasi- } \\
\text { experimental }\end{array}$ & AMC & $\begin{array}{l}\text { pediatric patients } \\
\text { and families }\end{array}$ & $\begin{array}{l}\text { preintervention }=70 \\
\text { postintervention }=41\end{array}$ & $\begin{array}{l}\text { Multifaceted interprofessional } \\
\text { education and collaborative project }\end{array}$ \\
\hline $\begin{array}{l}\text { Southwick et } \\
\text { al }(2014)^{40}\end{array}$ & $\begin{array}{l}\text { quasi- } \\
\text { experimental }\end{array}$ & AMC & $\begin{array}{l}\text { hospitalized } \\
\text { patients on } \\
\text { medicine resident } \\
\text { team }\end{array}$ & $\begin{array}{l}\text { control }=44 \\
\text { intervention }=46\end{array}$ & Multidisciplinary rounds \\
\hline $\begin{array}{l}\text { Townsend- } \\
\text { Gervis et al } \\
(2014)^{41}\end{array}$ & $\begin{array}{l}\text { quasi- } \\
\text { experimental }\end{array}$ & $\begin{array}{l}\text { suburban } \\
\text { hospital }\end{array}$ & $\begin{array}{l}\text { medical/surgical } \\
\text { unit patients }\end{array}$ & not provided & $\begin{array}{l}\text { Structured communication tool (SBAR) } \\
\text { and interdisciplinary rounds }\end{array}$ \\
\hline $\begin{array}{l}\text { Wray et al } \\
(2016)^{42}\end{array}$ & $\begin{array}{l}\text { quasi- } \\
\text { experimental }\end{array}$ & AMC & $\begin{array}{l}\text { general medicine } \\
\text { patients on resident } \\
\text { and nonteaching } \\
\text { services }\end{array}$ & $\begin{array}{l}\text { general medicine }= \\
4591 \\
\text { hospitalist }=1811\end{array}$ & $\begin{array}{l}\text { Physician-only team (general medicine } \\
\text { team) vs interprofessional dyad } \\
\text { (hospitalist-NP/PA dyad) }\end{array}$ \\
\hline
\end{tabular}

$A M C$, academic medical center; RCT, randomized controlled trial; MLP, midlevel practitioner; NP/PA, nurse practitioner/ physician assistant. 
Table 2. Team Composition and Intervention

\begin{tabular}{|c|c|c|c|}
\hline Study & Team Matrix & Team Category & Team Intervention \\
\hline Ahmed et al $(2012)^{22}$ & $\begin{array}{l}\text { geriatrician, NP, nurse, patient care assistant, } \\
\text { unit clerk, clinical nurse specialist; part- } \\
\text { time participation from pharmacist, physical } \\
\text { therapist, occupational therapist, social worker, } \\
\text { case manager }\end{array}$ & multiprofessional & teamwork \\
\hline Arbaje et al $(2010)^{27}$ & geriatrician, geriatric NP & dyad & teamwork \\
\hline Auerbach et al $(2011)^{30}$ & $\begin{array}{l}\text { physician, nurse, pharmacist (unit-based or } \\
\text { central), staff, patient }\end{array}$ & multiprofessional & coordination \\
\hline DeBehnke et al $(2002)^{31}$ & physician, nurse & dyad & teamwork \\
\hline Finch et al $(1999)^{23}$ & $\begin{array}{l}\text { physician, social worker, utilization manager, } \\
\text { dietician, pharmacist, respiratory therapist }\end{array}$ & multiprofessional & coordination \\
\hline Forster et al $(2005)^{25}$ & $\begin{array}{l}\text { physician, residents, staff internist, clinical } \\
\text { nurse specialist }\end{array}$ & dyad & collaboration \\
\hline Gade et al $(2008)^{28}$ & $\begin{array}{l}\text { physician (palliative care specialist), nurse, } \\
\text { social worker, chaplain }\end{array}$ & multiprofessional & teamwork \\
\hline Hastings et al $(2016)^{26}$ & physicians, nurses, allied health staff, family & multiprofessional & coordination \\
\hline Hung et al $(2013)^{32}$ & $\begin{array}{l}\text { geriatrician, geriatrics fellow, social worker, } \\
\text { clinical nurse specialist }\end{array}$ & multiprofessional & teamwork \\
\hline lannuzzi et al $(2014)^{33}$ & $\begin{array}{l}\text { hospitalist physician, resident, interns, students, } \\
\text { an NP or a PA }\end{array}$ & dyad & teamwork \\
\hline Kane et al $(2016)^{34}$ & $\begin{array}{l}\text { physician, residents, case manager, social worker, } \\
\text { respiratory therapist pharmacy, nutritionist, nurse, } \\
\text { nurse manager, medical director }\end{array}$ & multiprofessional & coordination \\
\hline Kara et al $(2015)^{35}$ & $\begin{array}{l}\text { case manager, clinical nurse specialist, } \\
\text { pharmacist, nutritionist, hospitalist - all unit-based }\end{array}$ & multiprofessional & teamwork \\
\hline Laird-Fick et al $(2011)^{36}$ & physician (residents), nurses & dyad & collaboration \\
\hline San Martin-Rodriguez et al $(2008)^{37}$ & physicians (multispecialty), nurses & dyad & teamwork \\
\hline Menefee et al $(2014)^{38}$ & $\begin{array}{l}\text { physicians, care manager, social worker, } \\
\text { nutritionist, respiratory therapist, pharmacist, } \\
\text { patient care assistants, therapists }\end{array}$ & multiprofessional & teamwork \\
\hline Preen et al $(2005)^{24}$ & not specified but includes primary care provider & multiprofessional & collaboration \\
\hline Roy et al $(2008)^{39}$ & $\begin{array}{l}\text { hospitalist, PA, nurse, care coordinator, } \\
\text { pharmacist }\end{array}$ & multiprofessional & teamwork \\
\hline Scotten et al $(2015)^{29}$ & $\begin{array}{l}\text { physician, nurse, therapists, informaticists, } \\
\text { speech therapists }\end{array}$ & multiprofessional & coordination \\
\hline Southwick et al $(2014)^{40}$ & $\begin{array}{l}\text { physician, resident, pharmacist, nurse, case } \\
\text { manager }\end{array}$ & multiprofessional & coordination \\
\hline Townsend-Gervis et al $(2014)^{41}$ & $\begin{array}{l}\text { nurse, dieticians, pharmacists, social worker, } \\
\text { case managers, physician }\end{array}$ & multiprofessional & coordination \\
\hline Wray et al $(2016)^{42}$ & physician-NP/PA team vs physician-only teams & dyad & teamwork \\
\hline
\end{tabular}

$N P$, nurse practitioner; $P A$, physician assistant.

\section{Study Findings}

The primary aim of this review was to determine the relationship between team-based models of care and patient satisfaction in the hospital setting. Collectively, $57 \%$ of the 21 studies $(n=12)$ found a significant improvement in patient satisfaction with the implementation of team-based care. Additionally,
5 studies found improved but not statistically significant patient satisfaction scores with teambased models of care $(81 \%, n=17)$ (Table 3$)$. In studies that examined other outcomes as their primary measure(s), patient satisfaction was observed as a secondary outcome and continued to demonstrate improvement. $^{22,34,41}$ 
Table 3. Study Results

\begin{tabular}{|c|c|c|c|}
\hline Citation & $\begin{array}{l}\text { Patient Satisfaction } \\
\text { Instrument }\end{array}$ & $\begin{array}{l}\text { Patient Satisfaction } \\
\text { Outcome }\end{array}$ & Other Outcomes \\
\hline Ahmed et al $(2012)^{22}$ & HCAHPS, Press Ganey & $\begin{array}{l}\text { Press Ganey scores remained } \\
\text { greater than } 80 \% \\
\text { HCAHPS scores consistently } \\
9 \text { or } 10 \\
\end{array}$ & $\begin{array}{l}\text { LOS, CMI-adjusted LOS, } \\
\text { direct costs, and readmission } \\
\text { decreased; CMI increased }\end{array}$ \\
\hline Arbaje et al $(2010)^{27}$ & $\begin{array}{l}\text { 4-question in-house survey } \\
\text { with 5-point Likert scale (not } \\
\text { validated) }\end{array}$ & $\begin{array}{l}\text { Slightly improved }(95.2 \% \text { vs } \\
93.8 \%, P=0.21)\end{array}$ & $\begin{array}{l}\text { CTM }=\text { NS }(\beta \text { coefficient: } 1.81 \\
P=0.47)\end{array}$ \\
\hline Auerbach et al $(2011)^{30}$ & $\begin{array}{l}\text { HCAHPS and in-house } \\
\text { questions }\end{array}$ & $\begin{array}{l}\text { Improved patient satisfaction } \\
\text { scores }(P<0.05)\end{array}$ & $\begin{array}{l}\text { LOS and readmission = NS } \\
(\mathrm{P}=0.78 \text { and } \mathrm{P}=0.09) \text {; care } \\
\text { perception = varied; quality of } \\
\text { teamwork = varied }\end{array}$ \\
\hline DeBehnke et al $(2002)^{31}$ & Press Ganey/HCAHPS & $\begin{array}{l}\text { Improved from } 78.2 \pm 20.4 \% \text { to } \\
82.2 \pm 17.2 \%(P<0.01)\end{array}$ & $\begin{array}{l}\text { Perception of waiting time and } \\
\text { staff attention improved }\end{array}$ \\
\hline Finch et al $(1999)^{23}$ & 11-question in-house survey & $\begin{array}{l}\text { Improved from } \sim 82 \% \text { to } \\
\sim 97 \% \text { and from Very Good to } \\
\text { Excellent }\end{array}$ & $\mathrm{N} / \mathrm{A}$ \\
\hline Forster et al $(2005)^{25}$ & $\begin{array}{l}\text { National Patient Satisfaction } \\
\text { Survey (Canadian) }\end{array}$ & Improved: 8.2 vs $7.6(\mathrm{P}=0.052)$ & $\begin{array}{l}\text { NS for in-hospital and } \\
\text { posthospital adverse events } \\
(P=0.10 \text { to } 0.87) \text {, readmission } \\
(P=0.16) \text {, return to ED }(P=0.10) \text {, } \\
\text { and death }(P=0.89)\end{array}$ \\
\hline Gade et al $(2008)^{28}$ & $\begin{array}{l}\text { Modified City of Hope Patient } \\
\text { Questionnaire: Place of Care } \\
\text { Environment scale, Doctors, } \\
\text { Nurses/OHCP Communication } \\
\text { scale }\end{array}$ & $\begin{array}{l}\text { Improved: } 6.8 \text { vs } 6.4(P<0.001) \\
\text { for facility, and } 8.3 \text { vs } 7.2 \\
(P<0.001) \text { for communication }\end{array}$ & $\begin{array}{l}\text { Quality of life, survival, and } \\
\text { advance directives = NS } \\
(P=0.10 \text { to } 0.80) ; \text { decreased } \\
\text { cost }(\$ 6766 \text { savings per patient }) \\
(P=0.001)\end{array}$ \\
\hline Hastings et al $(2016)^{26}$ & $\begin{array}{l}\text { Validated Canadian Patient } \\
\text { Experiences Survey - inpatient } \\
\text { care }\end{array}$ & $\begin{array}{l}\text { Family satisfaction improved } \\
\text { to } 58 \% \text { from } 50 \% \text {, as did } \\
\text { medication information (to } 95 \% \\
\text { from } 56 \% \text { ) }\end{array}$ & $\begin{array}{l}\text { Slightly decreased 30-day } \\
\text { readmission rates. }\end{array}$ \\
\hline Hung et al $(2013)^{32}$ & CTM, HCAHPS & $\begin{array}{l}\text { Improved mean CTM: } 72.5 \\
(19.1 \mathrm{SD}) \text { vs } 64.9 \text { (16.5 SD) } \\
(\mathrm{P}<0.001) \\
\text { Improved HCAHPS: } 50 \% \text { vs } \\
44.1 \%(\mathrm{C})\end{array}$ & $\begin{array}{l}\text { Improved incidence of adverse } \\
\text { events and LOS; readmission = } \\
\text { NS (slightly improved) }\end{array}$ \\
\hline lanuzzi et al $(2015)^{33}$ & $\begin{array}{l}\text { HCAHPS questions CMS 6-8, } \\
\text { Press Ganey P1-5 scores for } \\
\text { physicians }\end{array}$ & $\begin{array}{l}\text { HCAHPS: resident team had } \\
\text { statistically higher scores } \\
\text { Press Ganey: NS ( } P=0.02 \text { to } \\
0.73)\end{array}$ & $\begin{array}{l}\text { For resident group: lower cost } \\
(P=0.57) \text {; lower LOS }(P<0.001) ; \\
\text { NS for mortality }(P=0.60) ; \text { and } \\
\text { decreased readmission rate } \\
(P=0.07)\end{array}$ \\
\hline Kane et al $(2016)^{34}$ & Press Ganey/HCAHPS & $\begin{array}{l}\text { Improved patient satisfaction } \\
\text { scores in two categories: } 67.1 \% \\
\text { to } 69.5 \% ; 78.9 \% \text { to } 79.4 \%\end{array}$ & $\begin{array}{l}\text { Improved discharges by noon } \\
\text { per day from } 14 \% \text { to } 24 \% \text {; } \\
\text { readmission decreased from } \\
11.3 \% \text { to } 11.2 \% \text { (P-value not } \\
\text { reported) }\end{array}$ \\
\hline Kara et al $(2015)^{35}$ & Interview rounds, HCAHPS & NS (P-value not reported) & $\begin{array}{l}\text { Increased ACT scores } \\
\text { associated with decreased } \\
\text { LOS and CMI-adjusted variable } \\
\text { direct cost }(P<0.001)\end{array}$ \\
\hline Laird-Fick et al $(2011)^{36}$ & $\begin{array}{l}\text { Validated } 25 \text {-item provider- } \\
\text { patient relationship } \\
\text { questionnaire }\end{array}$ & NS (P-value not reported) & $\begin{array}{l}\text { LOS, pain score, and } \\
\text { psychological treatment=NS }\end{array}$ \\
\hline $\begin{array}{l}\text { San Martin-Rodriguez } \\
\text { et al }(2008)^{37}\end{array}$ & $\begin{array}{l}\text { Press Ganey/HCAHPS inpatient } \\
\text { survey }\end{array}$ & Improved (4.50 vs 4.54) & $\begin{array}{l}\text { LOS, pain score, and } \\
\text { psychological treatment=NS. }\end{array}$ \\
\hline
\end{tabular}


Table 3 (cont). Study Results

\begin{tabular}{|c|c|c|c|}
\hline Citation & $\begin{array}{l}\text { Patient Satisfaction } \\
\text { Instrument }\end{array}$ & $\begin{array}{l}\text { Patient Satisfaction } \\
\text { Outcome }\end{array}$ & Other Outcomes \\
\hline Menefee et al $(2014)^{38}$ & $\begin{array}{l}\text { Value-based purchasing internal } \\
\text { dataset }\end{array}$ & Improved (+7.5\%) & $\begin{array}{l}\text { Decreased readmission rate } \\
\text { (-6); LOS unchanged between } \\
\text { groups }\end{array}$ \\
\hline Preen et al $(2005)^{24}$ & In-house survey, not validated & $\begin{array}{l}\text { Improved (36.5\% improved, } \\
\mathrm{P}=0.02)\end{array}$ & N/A \\
\hline Roy et al $(2008)^{39}$ & $\begin{array}{l}\text { Press Ganey (include } \\
\text { HCAHPS) scores only } \\
\text { (physician scores and } \\
\text { discharge scores) }\end{array}$ & $\begin{array}{l}\text { NS between groups (P-value } \\
\text { not reported) }\end{array}$ & $\begin{array}{l}\text { Intervention group = higher } \\
\text { LOS }(95 \% \mathrm{CI} 95 \% \text { : }-0.4 \text { to } \\
10) \text {, lower costs }(95 \% \mathrm{Cl} \text { : } \\
-7.5 \text { to }-0.3 \%) \text {; NS for } 30 \text {-day } \\
\text { readmission }\end{array}$ \\
\hline Scotten et al $(2015)^{29}$ & $\begin{array}{l}\text { Engagement of Healthcare } \\
\text { Provider Services }\end{array}$ & NS (90.84 vs 90.08; $P=0.69)$ & $\begin{array}{l}\text { LOS = NS; readmission rates } \\
\text { increased ( } 7.45 \text { vs } 12.18 \\
\mathrm{P}=0.005)\end{array}$ \\
\hline Southwick et al $(2014)^{40}$ & Self-made survey & $\begin{array}{l}\text { NS }(P=0.076) \text {, intervention } 4.3 \\
\text { vs control } 4.0 \text { on Likert scale }\end{array}$ & $\begin{array}{l}\text { Improved LOS and 30-day } \\
\text { readmission scores }\end{array}$ \\
\hline $\begin{array}{l}\text { Townsend-Gervis et al } \\
(2014)^{41}\end{array}$ & HCAHPS & Slightly improved (72\% to $80 \%)$ & $\begin{array}{l}\text { Improved Foley catheter } \\
\text { removal }(P<0.001) \text { and } \\
\text { readmission rates }(P<0.001)\end{array}$ \\
\hline Wray et al $(2016)^{42}$ & $\begin{array}{l}\text { Picker-Commonwealth Survey/ } \\
\text { HCAHPS }\end{array}$ & $\begin{array}{l}\text { Improved }(73 \% \text { vs } 68 \%, \\
P=0.001)\end{array}$ & $\begin{array}{l}\text { Patient satisfaction scores only } \\
\text { were examined; no quality } \\
\text { metrics to compare; median } \\
\text { LOS similar between groups }\end{array}$ \\
\hline
\end{tabular}

ACT, accountable care teams; CMI, case mix index; CTM, care transitions measure; ED, emergency department; HCAHPS, Hospital Consumer Assessment of Healthcare Providers and Systems; LOS, length of stay; OHCP; Other Health Care Providers; NS, not statistically significant; SD, standard deviation.

Studies with improved patient satisfaction were more likely to utilize multiprofessional $(n=8,67 \%)$ versus dyad $(n=4,33 \%)$ teams. More than half of the team interventions were categorized as interprofessional teamwork $(52 \%, \mathrm{n}=11)$. Within this group, 6 teams were multiprofessional and 5 were dyads (Table 4). Seven studies reported team interventions consistent with interprofessional coordination (33\%), all of which were composed of multiprofessional teams. Based on team intervention analysis, studies demonstrating improved patient satisfaction more often incorporated interprofessional teamwork $(n=6$, $50 \%$ ) than studies that incorporated interprofessional coordination $(n=4,33 \%)$ or interprofessional collaboration $(n=2,17 \%)$.

In addition to examining team composition and intervention type as secondary outcomes, the relationship between patient satisfaction and other quality outcomes was analyzed. The majority of studies reviewed $(\mathrm{n}=18)$ measured both quality outcomes (length of stay, adverse events in the hospital, cost, 30-day readmission rate) and patient satisfaction (Table 3). Of the 21 total studies, 5 (24\%) showed significant improvement in both patient satisfaction and 1 or more other quality outcomes; $6(29 \%)$ demonstrated improvement in at least 1 quality outcome but did not demonstrate improved patient satisfaction; and 5 found improved patient satisfaction without improvements in other outcomes. The remaining 2 studies did not demonstrate improvements in either category (Table 5).

\section{DISCUSSION}

We reviewed existing literature to examine the relationship between team-based care and patient satisfaction. Most studies (57\%) demonstrated an improvement in patient satisfaction scores associated with team-based care. Studies that utilized multiprofessional teams (vs dyads) and an interprofessional teamwork intervention were more likely to demonstrate improved patient 
Table 4. Team Interventions and Impact on Patient Satisfaction

\begin{tabular}{|c|c|c|c|}
\hline & $\begin{array}{l}\text { Interprofessional } \\
\text { Coordination }\end{array}$ & $\begin{array}{l}\text { Interprofessional } \\
\text { Collaboration }\end{array}$ & $\begin{array}{l}\text { Interprofessional } \\
\text { Teamwork }\end{array}$ \\
\hline \multirow{6}{*}{$\begin{array}{l}\text { Improved patient } \\
\text { satisfaction }\end{array}$} & Auerbach et al $(2012)^{30}$ & ${ }^{*}$ Forster et al $(2005)^{25}$ & ${ }^{*}$ DeBehnke et al $(2002)^{31}$ \\
\hline & Finch et al $(1999)^{24}$ & Preen et al $(2005)^{24}$ & Gade et al $(2008)^{28}$ \\
\hline & Hastings et al $(2016)^{26}$ & & Hung et al $(2013)^{32}$ \\
\hline & Kane et al $(2016)^{34}$ & & Menefee et al $(2014)^{38}$ \\
\hline & & & ${ }^{*}$ San Martin-Rodriguez et al $(2008)^{37}$ \\
\hline & & & *Wray et al $(2016)^{42}$ \\
\hline \multirow{5}{*}{$\begin{array}{l}\text { Did not improve } \\
\text { patient satisfaction }\end{array}$} & Scotten et al $(2015)^{29}$ & *Laird-Fick et al (2011) ${ }^{36}$ & Ahmed et al $(2012)^{22}$ \\
\hline & Southwick et al $(2014)^{40}$ & & ${ }^{*}$ Arbaje et al $(2010)^{27}$ \\
\hline & Townsend-Gervis et al $(2014)^{41}$ & & Kara et al $(2015)^{36}$ \\
\hline & & & *lanuzzi et al (2015) ${ }^{33}$ \\
\hline & & & Roy et al $(2008)^{39}$ \\
\hline
\end{tabular}

*Indicates dyad teams; no asterisk indicates multiprofessional teams.

Table 5. Quality Metrics and Patient Satisfaction

\begin{tabular}{|c|c|c|}
\hline & Improved Patient Satisfaction & No Improved Patient Satisfaction \\
\hline \multirow[t]{6}{*}{ Improved quality metrics(s)* } & Gade et al $(2008)^{28}$ & Ahmed et al $(2012)^{22}$ \\
\hline & Hung et al $(2013)^{32}$ & lanuzzi et al $(2015)^{33}$ \\
\hline & Kane et al $(2016)^{34}$ & Kara et al $(2015)^{35}$ \\
\hline & Menefee et al $(2014)^{38}$ & Roy et al $(2008)^{39}$ \\
\hline & San Martin-Rodriguez et al $(2008)^{37}$ & Southwick et al $(2014)^{40}$ \\
\hline & & Townsend-Gervis et al $(2014)^{41}$ \\
\hline \multirow[t]{7}{*}{ No improved quality metrics(s) } & Auerbach et al $(2012)^{30}$ & ${ }^{* *}$ Arbaje et al $(2010)^{27}$ \\
\hline & ${ }^{* *}$ DeBehnke et al $(2002)^{31}$ & Laird-Fick et al (2011) \\
\hline & ${ }^{* *}$ Finch et al $(1999)^{23}$ & Scotten et al $(2015)^{29}$ \\
\hline & Forster et al $(2005)^{25}$ & \\
\hline & Hastings et al $(2016)^{26}$ & \\
\hline & Preen et al $(2005)^{24}$ & \\
\hline & Wray et al $(2016)^{42}$ & \\
\hline
\end{tabular}

*At least one quality metric improved in primary or secondary outcomes.

** Study did not evaluate quality metric.

satisfaction scores. Additionally, 52\% of the studies found improvement in at least 1 quality outcome in correlation with team-based care, but only $24 \%$ of the studies found improvement in both patient satisfaction and quality outcomes.

\section{Team-Based Care and Patient Satisfaction}

In a prior systematic review performed by Wen and Schulman, a slightly positive, but statistically insignificant, relationship was found between team-based care interventions and patient satisfaction, a measurement of the patient experience. ${ }^{13}$ Since 2012, when their 
research was conducted, many more studies including non-randomized control trials have been performed to analyze this question, which we have included herein.

Even more evidence found beyond the studies within this review supports team-based care, a culture that fosters teamwork, and their positive impact on the patient experience. Meterko et al studied the attributes of employees at 125 Veterans Administration hospitals and found a strong positive relationship between a culture of teamwork and patient satisfaction. They postulated that teamwork and a culture in which teams can thrive are the basis for patient satisfaction and an overall positive patient experience. ${ }^{14}$

\section{Team Composition and Patient Satisfaction}

To understand how teams can be designed to optimize patient satisfaction, we examined the team structure and types of interventions with the most impact on patient satisfaction. Team composition usually included a physician, nurse, and at least 1 other health profession. All but 1 of the 5 studies that showed improvement in both quality metrics and patient satisfaction utilized multiprofessional teams, often with greater than 4 different disciplines. Studies that found an improvement in quality metrics only also utilized multiprofessional teams more frequently than dyad teams ( 5 vs 1 , respectively). Works by Reeves et al and Fiscella et al recommend that larger, more diverse teams might have greater capacity to care for complex patients or provide a more comprehensive appearance to patients. ${ }^{8,43}$ While teamlets (physician and nurse or medical assistant) may have the ability to provide quality patient-centered care, multidisciplinary teams are better equipped to provide integrated and coordinated care for patients with complex chronic disease. ${ }^{43}$

\section{Team Interventions and Patient Satisfaction}

Equally important to the team structure is the process or intervention type. Studies that examined teambased models of care - those using a fully integrated and interdependent process to optimize patient care, described as interprofessional teamwork in the interprofessional literature - demonstrated improved patient satisfaction scores versus the other types of interventions. ${ }^{8}$ In their comprehensive review, Reeves et al provide a conceptual framework that describes examples in which teams function in a committed way for full integration of care and demonstrate improved patient outcomes and improved team satisfaction. ${ }^{8}$ In previous studies, team effectiveness was linked with integrated, cross-functional teams that coalesce to create their own identity. ${ }^{44}$ According to Lemieux-Charles and McGuire, redesigned care teams with full integration were more likely to positively impact patient outcomes than usual team care. ${ }^{45}$ This calls for further studies on fully integrated interprofessional teamwork and its effect on patient outcomes, especially patient satisfaction.

An equivocal relationship between care quality outcomes and patient satisfaction has been documented. ${ }^{16,46,47}$ Both are linked to reimbursement under the pay-for-performance guidelines and should be considered for promotion of high-value patient-centered care. In our review, team-based care was associated with both patient satisfaction and quality patient outcomes, but the association between improved quality and patient satisfaction was inconsistent (Table 4). Only 5 studies demonstrated improved quality metrics and patient satisfaction. In contrast, a study by Anhang et al did find a strong relationship between hospitals who reported high patient satisfaction scores (as measured by HCAHPS) and quality outcomes, primarily acute myocardial infarction, heart failure, pneumonia, and surgery. ${ }^{46}$ However, in a similar study by Lyu et al, patient satisfaction scores were not statistically correlated with quality outcomes in 31 hospitals, and the authors concluded patient satisfaction is an independent measure and not codependent on quality outcomes. ${ }^{47}$ The discrepancy in the literature is supported by the findings of this review and implores for more research in this area.

\section{The Link to Patient Experience}

There are many potential reasons for the lack of association between the patient's perception of care and the measured outcomes. Patient satisfaction can be very subjective and is confounded by many factors outside of specific quality indicators. ${ }^{47}$ Additionally, HCAHPS has been challenged as an inaccurate measurement to account for the numerous factors that contribute to the patient experience, such as teamwork..$^{16,47}$

One explanation could be related to the degree to which the patient is engaged in their care by the health 
care team. Patient activation, a component of patient engagement, is defined as the patient's ability to understand their care and have the knowledge, skills, and confidence to navigate their own care. ${ }^{48}$ Extensive research performed by Hibbard et al demonstrated improved patient outcomes, lower health care costs and higher patient experience scores when patients are highly activated. ${ }^{48,49}$ Patient activation may provide the missing link between patient satisfaction and improved quality. A highly activated patient may perceive they are part of their medical care team and lead to improved patient experiences, thus diminishing the disparity between the patient's perception of quality and actual quality metrics. For example, in this review, both studies that captured the patient's perspective (ie, patient included as team member, family/ caregiver involvement, patient perception of team) demonstrated both improved patient satisfaction scores and improved (though not statistically significant) quality metric outcomes. ${ }^{26,30}$ Both of these studies that included the patient as part of the team had similar team interventions: interprofessional coordination. This type of intervention is considered a lower level of integration and patient activation and could account for the marginal significance statistically.

Although significant correlational research has been conducted examining patient activation and patient experience, more studies are needed to explore a causal relationship between the degree of patient activation, patient satisfaction, and quality outcomes to fully understand the elements of the patient experience.

\section{Limitations and Bias}

This review included articles with various study designs and did not limit inclusion criteria to randomized controlled trials. The heterogeneity of designs led to the low to moderate quality assessment of the articles. This may have introduced bias and is a limitation of this review.

Another limitation of the review was the predominant use of HCAHPS to measure patient satisfaction. Although HCAHPS is the primary tool used to measure patient satisfaction posthospitalization in the United States, its effectiveness has been questioned because of the many other factors affecting patient satisfaction outside of the provider's control. ${ }^{50}$ In our review, 4 of the 5 studies demonstrating an association between improved quality metrics and patient satisfaction utilized HCAHPS. However, it is difficult to quantify a relationship between care provided by the medical care team and patient satisfaction, as HCAHPS does not assess the whole team, only the nurse and physician providing care. ${ }^{15} \mathrm{~A}$ more rigorous and comprehensive measurement of the patient experience should include questions about the care team and the attribution the team has on patient satisfaction scores.

\section{CONCLUSIONS}

To date, team-based care has demonstrated a positive effect on the health care system and contributes to high-value, patient-centered care. Patient satisfaction is emerging as an important indicator of quality, increasingly linked to reimbursement under the new pay-for-performance payment models. In reviewing reported relationships between team-based care in the hospital setting and patient satisfaction, we found that studies utilizing team-based care had improved patient satisfaction. Team composition and type of team-based intervention, potentially including the patient, may play an important role in patient satisfaction outcomes.

The relationship between other quality outcomes (eg, length of stay, adverse events, hospital readmission) and patient satisfaction appears dichotomous, given that many articles found improved patient satisfaction scores did not always have improved quality metric outcomes in parallel. This observation uncovers a gap in research: the patient's perception of quality does not necessarily match how the health care industry perceives quality. More research should be performed to identify the relationship between the patient experience, team composition, and type of team interventions to identify optimal team based-care models and inform clinical practice guidelines, health care policy, and reimbursement for team-based models of care.

Additionally, the manner in which patient satisfaction is measured and reported as a means for reimbursement should be further analyzed. Current assessments of patient satisfaction do not account for all members of the health care team and therefore do not reflect the impact that team-based models of care have on the patient experience. Through the 
impact of outcomes-driven research, development of new interprofessional training programs, teambased clinical practice guidelines, and health policy promoting reimbursement for team care, the overall patient experience can be transformed.

\section{Patient-Friendly Recap}

-While use of team-based care approaches is increasing, their ultimate effect on patient satisfaction is unknown.

- The authors reviewed reported studies for methods and results concerning composition of teams, types of intervention, patient satisfaction scores, and outcomes for patients cared for in a hospital.

- They found that health care teams that represented more than two professions, worked closely together, and could address multiple patient issues yielded greater improvement in patient satisfacton.

\section{Author Contributions}

Study design: Will, Lamb. Data acquisition or analysis: Will, Johnson. Manuscript drafting: all authors. Critical revision: Will, Lamb.

\section{Conflicts of Interest}

None.

\section{References}

1. Berwick DM, Nolan TW, Whittington J. The triple aim: care, health, and cost. Health Aff (Millwood). 2008;27:759-69. $\underline{\text { CrossRef }}$

2. Mitchell P, Wynia M, Golden R, et al. Core principles \& values of effective team-based health care. Published October 2012. https://nam.edu/wp-content/uploads/2015/06/VSRT-TeamBased-Care-Principles-Values.pdf. Washington, DC: Institute of Medicine. Accessed February 20, 2018.

3. Green BN, Johnson CD. Interprofessional collaboration in research, education, and clinical practice: working together for a better future. J Chiropr Educ. 2015;29:1-10. CrossRef

4. Institute of Medicine (US) Committee on the Robert Wood Johnson Foundation Initiative on the Future of Nursing. The Future of Nursing: Leading Change, Advancing Health. Washington, DC: National Academies Press, 2011. CrossRef

5. Naylor MD, Coburn KD, Kurtzman ET, et al. Inter-professional team-based primary care for chronically ill adults: state of the science. Unpublished white paper presented at the ABIM Foundation Meeting to Advance Team-Based Care for the Chronically Ill in Ambulatory Settings, Philadelphia, PA, March 24-25, 2010.
6. Department of Human Resources for Health. Framework for Action on Interprofessional Education \& Collaborative Practice. Geneva, Switzerland: World Health Organization, 2010. www.who.int/hrh/resources/framework_action/en. Accessed September 2018.

7. Interprofessional Education Collaborative. Core Competencies for Interprofessional Collaborative Practice: 2016 Update. Washington, DC: Interprofessional Education Collaborative, 2016.

8. Reeves S, Lewin S, Espin S, Zwarenstein M. Interprofessional Teamwork for Health and Social Care. Chichester, United Kingdom: Blackwell Publishing, 2010.

9. Bodenheimer T. Building Teams in Primary Care: Lessons Learned. Oakland, CA: California HealthCare Foundation, 2007.

10. Brandt B, Lutfiyya MN, King JA, Chioreso C. A scoping review of interprofessional collaborative practice and education using the lens of the Triple Aim. J Interprof Care. 2014;28:393-9. CrossRef

11. Reeves S, Perrier L, Goldman J, Freeth D, Zwarenstein M. Interprofessional education: effects on professional practice and healthcare outcomes (update). Cochrane Database Syst Rev. 2013;28(3):CD002213. CrossRef

12. Salzberg CA, Bitton A, Lipsitz SR, et al. The impact of alternative payment in chronically ill and older patients in the patientcentered medical home. Med Care. 2017;55:483-92. CrossRef

13. Wen J, Schulman KA. Can team-based care improve patient satisfaction? A systematic review of randomized controlled trials. PLoS One. 2014;9(7):e100603. CrossRef

14. Meterko M, Mohr DC, Young GJ. Teamwork culture and patient satisfaction in hospitals. Med Care. 2004;42:492-8. CrossRef

15. Hospital Consumer Assessment of Healthcare Providers and Systems. CAHPS ${ }^{\circledR}$ Hospital Survey. Last modified 2019 Mar 22. http://www.hcahpsonline.org. Accessed March 25, 2019.

16. Manary MP, Boulding W, Staelin R, Glickman SW. The patient experience and health outcomes. $N$ Engl J Med. 2013;368:201-3. CrossRef

17. Jha AK, Orav EJ, Zheng J, Epstein AM. Patients' perception of hospital care in the United States. $N$ Engl $J$ Med. 2008;359:1921-31. CrossRef

18. Moher D, Liberati A, Tetzlaff J, Altman DG; PRISMA Group. Preferred reporting items for systematic reviews and meta-analyses: the PRISMA statement. PLoS Med. 2009;6(7):e1000097. CrossRef

19. Grant MJ, Booth A. A typology of reviews: an analysis of 14 review types and associated methodologies. Health Info Libr J. 2009;26:91-108. CrossRef

20. Harbour R, Miller J. A new system for grading recommendations in evidence based guidelines. BMJ. 2001;323:334-6. CrossRef

21. Fleiss JL, Levin B, Paik MC. The measurement of interrater agreement. In: Statistical Methods for Rates and Proportions, Third Edition. Hoboken, NJ: John Wiley \& Sons, 2003, pp. 598-626. CrossRef

22. Ahmed N, Taylor K, McDaniel Y, Dyer CB. The role of an Acute Care for the Elderly unit in achieving hospital quality indicators while caring for frail hospitalized elders. Popul Health Manag. 2012;15:236-40. CrossRef

23. Finch GL, Linderbery J. Improving patient satisfaction through unit-based team case management. Continuum. 1999;19(2):12-6.

24. Preen DB, Bailey BE, Wright A, et al. Effects of a multidisciplinary, post-discharge continuance of care intervention on quality of life, discharge satisfaction, and hospital length of stay: a randomized controlled trial. Int $J$ Qual Health Care. 2015;17:43-51. CrossRef 
25. Forster AJ, Clark HD, Menard A, et al. Effect of a nurse team coordinator on outcomes for hospitalized medicine patients. Am J Med. 2005;118:1148-53. CrossRef

26. Hastings SE, Suter E, Bloom J, Sharma K. Introduction of a team-based care model in a general medical unit. BMC Health Serv Res. 2016;16:245. CrossRef

27. Arbaje AI, Maron DD, Yu Q, et al. The geriatric floating interdisciplinary transition team. $J \mathrm{Am}$ Geriatr Soc. 2010;58:364-70. CrossRef

28. Gade G, Venohr I, Conner D, et al. Impact of an inpatient palliative care team: a randomized control trial. J Palliat Med. 2008;11:180-90. CrossRef

29. Scotten M, Manos EL, Malicoat A, Paolo AM. Minding the gap: interprofessional communication during inpatient and post discharge chasm care. Patient Educ Couns. 2015;98:895900. CrossRef

30. Auerbach AD, Sehgal NL, Blegen MA, et al. Effects of a multicentre teamwork and communication programme on patient outcomes: results from the Triad for Optimal Patient Safety (TOPS) project. BMJ Qual Saf. 2012;21:118-26. CrossRef

31. DeBehnke D, Decker MC. The effects of a physician-nurse patient care team on patient satisfaction in an academic ED. Am J Emerg Med. 2002;20:267-70. CrossRef

32. Hung WW, Ross JS, Farber J, Siu AL. Evaluation of the Mobile Acute Care of the Elderly (MACE) service. JAMA Intern Med. 2013;173:990-6. CrossRef

33. Iannuzzi MC, Iannuzzi JC, Holtsbery A, Wright SM, Knohl SJ. Comparing hospitalist-resident to hospitalist-midlevel practitioner team performance on length of stay and direct patient care cost. J Grad Med Educ. 2015;7:65-9. CrossRef

34. Kane M, Weinacker A, Arthofer R, et al. A multidisciplinary initiative to increase inpatient discharges before noon. J Nurs Admin. 2016;46:630-5.

35. Kara A, Johnson CS, Nicley A, Niemeier MR, Hui SL. Redesigning inpatient care: testing the effectiveness of an accountable care team model. J Hosp Med. 2015;10:773-9. CrossRef

36. Laird-Fick HS, Solomon D, Jodoin C, et al. Training residents and nurses to work as a patient-centered care team on a medical ward. Patient Educ Couns. 2011;84:90-7. CrossRef

37. San Martin-Rodriguez L, D'Amour D, Leduc N. Outcomes of interprofessional collaboration for hospitalized cancer patients. Cancer Nurs. 2008;31(2):E18-E27. CrossRef

38. Menefee KS. The Menefee model for patient-focused interdisciplinary team collaboration. $J$ Nurs Admin. 2014;44:598-605. CrossRef
39. Roy CL, Liang CL, Lund M, et al. Implementation of a physician assistant/hospitalist service in an academic medical center: impact on efficiency and patient outcomes. J Hosp Med. 2008;3:361-8. CrossRef

40. Southwick F, Lewis M, Treloar D. Applying athletic principles to medical rounds to improve teaching and patient care. Acad Med. 2014;89:1018-23. CrossRef

41. Townsend-Gervis M, Cornell P, Vardaman JM. Interdisciplinary rounds and structured communication reduce re-admissions and improve some patient outcomes. West J Nurs Res. 2014;36:917-28. CrossRef

42. Wray CM, Flores A, Padula WV, Prochaska MT, Meltzer DO, Arora VM. Measuring patient experiences on hospitalist and teaching services: patient responses to a 30-day postdischarge questionnaire. J Hosp Med. 2016;11:99-104. CrossRef

43. Fiscella K, Mauksch L, Bodenheimer T, Salas E. Improving care teams' functioning: recommendations from team science. Jt Comm J Qual Patient Saf. 2017;43:361-8. CrossRef

44. Wensing M, Wollersheim H, Grol R. Organizational interventions to implement improvements in patient care: a structured review of reviews. Implement Sci. 2006;1:2. CrossRef

45. Lemieux-Charles L, McGuire WL. What do we know about health care team effectiveness? A review of the literature. Med Care Res Rev. 2006;63:263-300. CrossRef

46. Anhang Price R, Elliott M, Zaslavsky A. Examining the role of patient experience surveys in measuring health care quality. Med Care Res Rev. 2014;71:522-54. CrossRef

47. Lyu H, Wick EC, Housman M, Freischlag JA, Makary MA. Patient satisfaction as a possible indicator of quality surgical care. JAMA Surg. 2013;148;362-7. CrossRef

48. Hibbard JH, Greene J, Overton V. Patients with lower activation associated with higher costs; delivery systems should know their patients' 'scores.' Health Aff (Millwood). 2013;32:216-22. CrossRef

49. Greene J, Hibbard JH, Sacks RM, Overton V. Understanding the relationship between patient engagement and patient experiences of care. Unpublished paper. 2013.

50. Roland M, Elliott M, Lyratzopoulos G, et al. Reliability of patient responses in pay for performance schemes: analysis of national General Practitioner Patient Survey data in England. BMJ. 2009;339:b3851. CrossRef

(C) 2019 Aurora Health Care, Inc. 